Research Article

\title{
What does determine the sign of core in Magnetic Flux Rope structures of the Earth's magnetotail
}

\author{
D. V.Sarafopoulos
}

Department of Electrical and Computer Engineering, Democritus University of Thrace, Xanthi, Greece

Received 30 September 2013; Accepted 10 September 2014

\begin{abstract}
This paper primarily examines the key factors being involved in precisely determining the sign of the core field in a magnetic flux rope (MFR) like structure embedded in the tailward plasma flow associated with the Earth's magnetotail. Magnetic flux ropes are frequently detected by satellites moving smoothly northwards (upwards) or southwards (downwards) and crossing almost the whole plasma sheet; the sign of the rope's core is associated with the local tail's motion: If the tail is bending to an upward or downward direction, then the sign of the rope's core, being essentially an intense $\mathrm{B}_{\mathrm{y}}$ deviation, will be positive or negative correspondingly. On the basis of this observational finding, a major question concerns the mechanism by which the tail's motion is dictated. The reconnection process acting in the tail will obviously produce symmetric structures of MFRs (with respect to the neutral sheet plane); therefore, the detected organized asymmetry may be an additional indication in the whole magnetotail' s dynamics. Moreover, we discuss the issue of the core's sign in cases without any significant magnetotail's motion. A model interpreting the diagnosed behavior is introduced: Once a tailward ion jet is produced in a thinned plasma sheet, it might form clockwise or counterclockwise ion vortices (i.e., loop-like ion currents) providing the "magnetic core" with the appropriate sign. The crucial role of the interplanetary $\mathrm{B}_{\mathrm{y}}$ deviation of the magnetic field (IMF) is scrutinized and taken into account. The whole model is tested under the condition of long-lasting extraordinary events characterized by a persistent-intense $\mathrm{B}_{\mathrm{y}}$ deviation with a duration up to $34 \mathrm{~min}$. This work, based on Geotail single-satellite measurements, is not a statistical one; it is a first approach allowing the reconstruction of measurements in the whole range of the magnetotail's deflections, from negligible up to stronger significant magnetotail movements, and should be therefore elucidated in more detail. The average duration for the studied ropes is $\sim 90 \mathrm{sec}$; they occur at distances greater than $25 \mathrm{R}_{\mathrm{E}}$ from the Earth.
\end{abstract}

Keywords: Plasma sheet, magnetic reconnection, magnetic flux rope, field-aligned currents, solar wind-magnetosphere interaction.

\section{Introduction}

From the perspective of the near Earth neutral line (NENL) model of magnetospheric substorms (e.g., [1]), the $\mathrm{B}_{\mathrm{y}}$ component of the interplanetary magnetic field (IMF) is usually considered that penetrates inside the magnetotail (e.g., [2] and references therein) and acts as a guide field around which a helical magnetic field is wrapped forming an MFR (e.g., [3, 4]). Throughout this work, we categorize a structure as being MFR-like with respect to the following morphological criteria: (a) The $\mathrm{B}_{\mathrm{y}}$ component of the magnetic field rapidly increases from its initial level to a peak value, forming "the core" of the supposed rope; (b) the $\mathrm{B}_{\mathrm{z}}$ component reverses its sign when the $\mathrm{B}_{\mathrm{y}}$ takes on its peak value showing a bipolar positive-then-negative signature; it may result from the helical magnetic field structure of rope; (c) the MFR is accompanied by tailward plasma flows, as it is anticipated for a "plasmoid flux rope"; this terminology appears, for instance, in the statistical works of Slavin et al.

\footnotetext{
* E-mail address: sarafo@ee.duth.gr
}

ISSN: 1791-2377 @ 2014 Kavala Institute of Technology. All rights reserved.
$[5,6]$, and (d) the total magnetic field- $B_{\text {total }}$ increases when the $B_{z}$ changes its sign. We have to stress that until today the simple concept for a direct interrelationship (via reconnection) between the sign of the rope's core in magnetotail and the sign of IMF- $\mathrm{B}_{\mathrm{y}}$ is prevailing. Is there any room for the catalytic action of another factor? Is there any element missing in the dynamics of today's magnetospheric physics at this point? We would like to incorporate a few new observational elements of crucial importance into the above "paradigm" that potentially lead to a more complete and deeper understanding.

One observation of this work, that may play a key role, is as follows: Frequently, when (and where) an MFR is detected, the magnetotail moves upward (northward) or downward (southward) and the kind of motion seems to be the critical factor determining the sign for the $B_{y}$ deviation. An upward motion generates a positive $\mathrm{B}_{\mathrm{y}}$ "core", whereas a negative $\mathrm{B}_{\mathrm{y}}$ "core" is originated by a downward motion. That is, an upward or downward local motion (i.e., when the magnetotail somewhere appropriately bends) produces a loop-like magnetic field structure encircling a single- 
compact $\mathrm{B}_{\mathrm{y}}$-core (i.e., a "one-core" magnetic field). What is dictating this kind of tail's motion? Is the motion the only one factor determining the rope's sign? What is then the specific role of the IMF- $B_{y}$ ? In addition, there are cases where the magnetotail appears motionless and the rope (consequently) remains symmetric around the X-axis; then inside the supposed closed structure a "two-core" magnetic field is expected to be formed: A positive $B_{y}$ deviation in the north and a negative one in the south plasma sheet. The "two-core" structure appears similar to a cross-tail "magnetic ring", a particularly spatial arrangement similar to the gas vortex rings called "smoke rings".

Sarafopoulos [7, 8] gives a few MFR-like events manifesting that strong intra-magnetospheric $B_{y}$ deviations are not directly driven by an exo-magnetosphere $B_{y}$ component. Most importantly, these events demonstrated that $\mathrm{B}_{\mathrm{y}}$ deviations inside the magnetotail have been simultaneously observed in various measurements by different CLUSTER satellites with opposite signs. Therefore, one has to reconcile the past results with the results set forth in this work, where the MFRs exclusively appear having a single and unique core. Presently, we are already able to make a particularly important distinction: In the just mentioned CLUSTER observations, the $\mathrm{B}_{\mathrm{y}}$ deviations last for a few seconds, whereas the typical MFR events last 1-2 min at distances 20-30 $\mathrm{R}_{\mathrm{E}}$ far away from the Earth; a differentiation that should be carefully taken into account. In this work, the studied MFR-like events are categorized as "typical"; they are similar to the majority of published ropes. A representative sample of "typical ropes" is presented in the statistical work of Slavin et al., [3]. This work mentions, even though parenthetically, some situations, where very strong and persistent positive IMF- $\mathrm{B}_{\mathrm{y}}$ values correspond, under certain circumstances, to persistent negative $B_{y}$ values in the magnetotail. The statistically established idea of the IMF- $B_{y}$ penetration in magnetotail do not prejudge that the intra-magnetosphere $B_{y}$ deviations should always be of the same sign with those of exo-magnetospheric origin, since the reality is much more sophisticated and multidimensional.

In this work, our approach is not a statistical one. We are going to establish our claims using indicative case studies. The decisive role of local bends (i.e., curvatures) in magnetotail is emphasized by six events; three with positive and three with negative $\mathrm{B}_{\mathrm{y}}$-core. A list of dates for a few additional events is simply cited. The situation for a locally motionless magnetotail is more complicated; we actually need at lest two satellites, one in the north and the other in the south plasma sheet. Nevertheless, in this work with exclusively Geotail datasets, we provide a preliminary evidence particularly useful for the discussion section. An interpretation mechanism is finally given that may reasonably answer the question concerning the $\mathrm{B}_{\mathrm{y}}$ sign in each sub-class; we are especially interested in that the proposed mechanism incorporates even the extraordinary events with long-lasting intra-magnetosphere $B_{y}$ deviations. Certainly a future statistical approach, in harmony with the presented selection criteria, would definitely substantiate our suggestions.

\section{Observations}

The below presented events are classified in four subcategories. The presentation format is the same for all the MFR-like events. The vector magnetic field is concurrently shown with the ion plasma velocity along the X-axis; the velocity is always directed tailward. The used magnetic field (in nanoteslas) is provided by the Geotail/MGF instrument [9], with $0.0625 \mathrm{sec}$ resolution time. The Geotail plasma data are generated by taking the moments over $12 \mathrm{sec}$ intervals of ion distribution functions measured by a low energy particle (LEP) instrument [10].

\subsection{MFRs occurring with (local) downward magnetotail motion}

In this subcategory, just three indicative events are selected and presented in Fig. 1; they occurred (a) on January 29, 1997 at 12:08:30 UT, (b) on February 05, 2000, at 22:57 UT and (c) on February 13, 1994, at 13:28 UT. In each case the Geotail smoothly moves from the south plasma sheet toward the north, crossing the neutral sheet; $\left|\Delta \mathrm{B}_{\mathrm{x}}\right|$ is greater than $12 \mathrm{nT}$ and the transition time is $2-5 \mathrm{~min}$. We discern four major negative $B_{y}$ deviations (marked with the capital letters A, B, C and D) associated with (a) bipolar positive-then-negative $B_{z}$ signatures, (b) total magnetic field increases and (c) tailward and steadily increasing ion flows. The third event with two well-separated MFR-like structures occurred in the distant tail, at $(\mathrm{X}, \mathrm{Y}, \mathrm{Z})_{\mathrm{GSM}}=(-65.2,9.1$, 2.3) $R_{E}$. For the first and second event the coordinates are ($29,-1.8,-2.7) R_{E}$ and $(-30.1,-4.6,-3.1) R_{E}$, respectively. Irrespectively of any $B_{x}$ micro-fluctuation, all the four $B_{y}$ deviations point dawnward.

\subsection{MFRs occurring with (local) upward magnetotail motion}

Each of the three MFR case studies presented in Fig. 2 demonstrates a positive $\mathrm{B}_{\mathrm{y}}$ deviation associated with a distinct bipolar $\mathrm{B}_{\mathrm{z}}$ signature, as previously in Fig. 1, plus a $\mathrm{B}_{\text {total }}$ increase and tailward ion flows. The Geotail smoothly moves from the northern hemisphere of plasma sheet toward the neutral sheet and finally crosses it; $\left|\Delta B_{x}\right| \cong 15$ $\mathrm{nT}$. The $\mathrm{B}_{\mathrm{y}}$ deviations marked as $\mathrm{E}, \mathrm{F}$ and $\mathrm{G}$ occurred at $(\mathrm{X}$, $\mathrm{Y}, \mathrm{Z})_{\mathrm{GSM}}=(-29,-8.4,-3) \mathrm{R}_{\mathrm{E}},(-28.4,1.7,4.9) \mathrm{R}_{\mathrm{E}}$ and $(-69.1$, $9.2,-0.5) R_{E}$, respectively. The ion velocity measurements are missing for the third case; however, the velocities in magnetotail beyond $\sim 60 \quad \mathrm{R}_{\mathrm{E}}$ are almost exclusively antisunward. Finally, it is not meaningless to stress that "the main finding" of this work is probably the outcome of this subsection. We mean that the demand for multi-satellite measurements is much higher in the next subsection. 


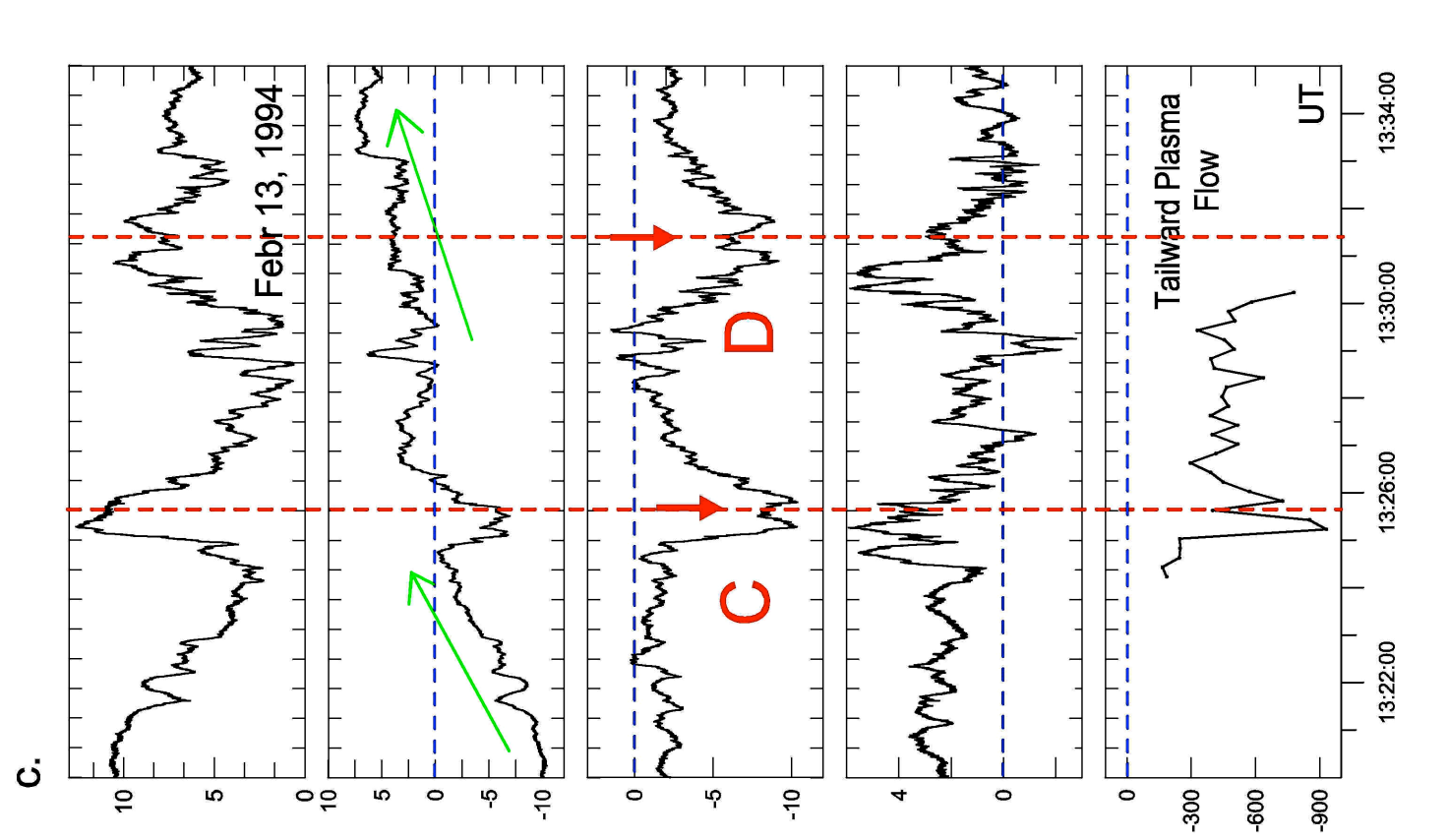

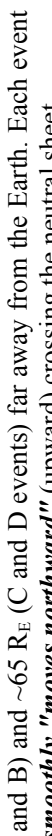

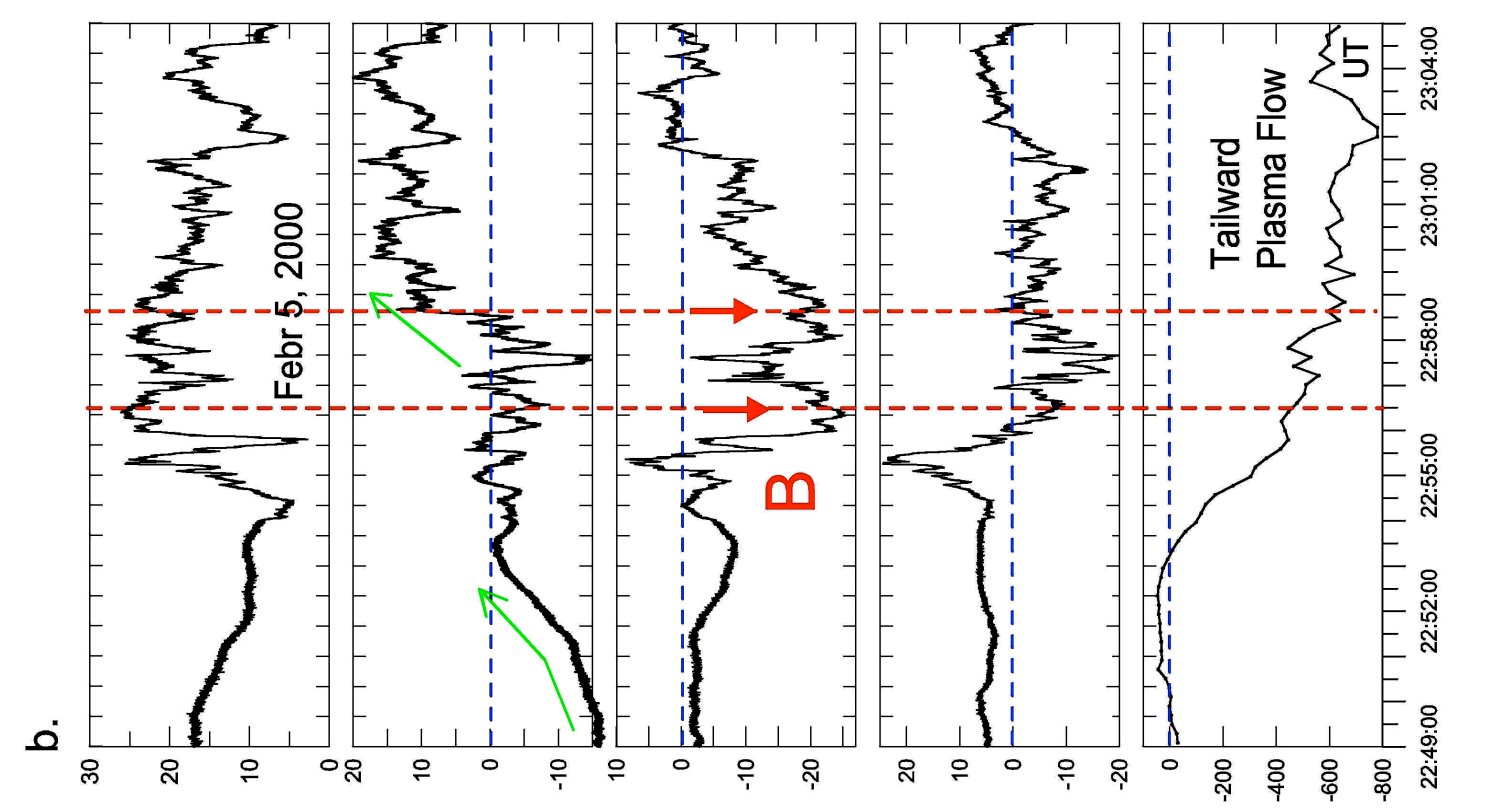

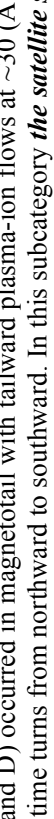

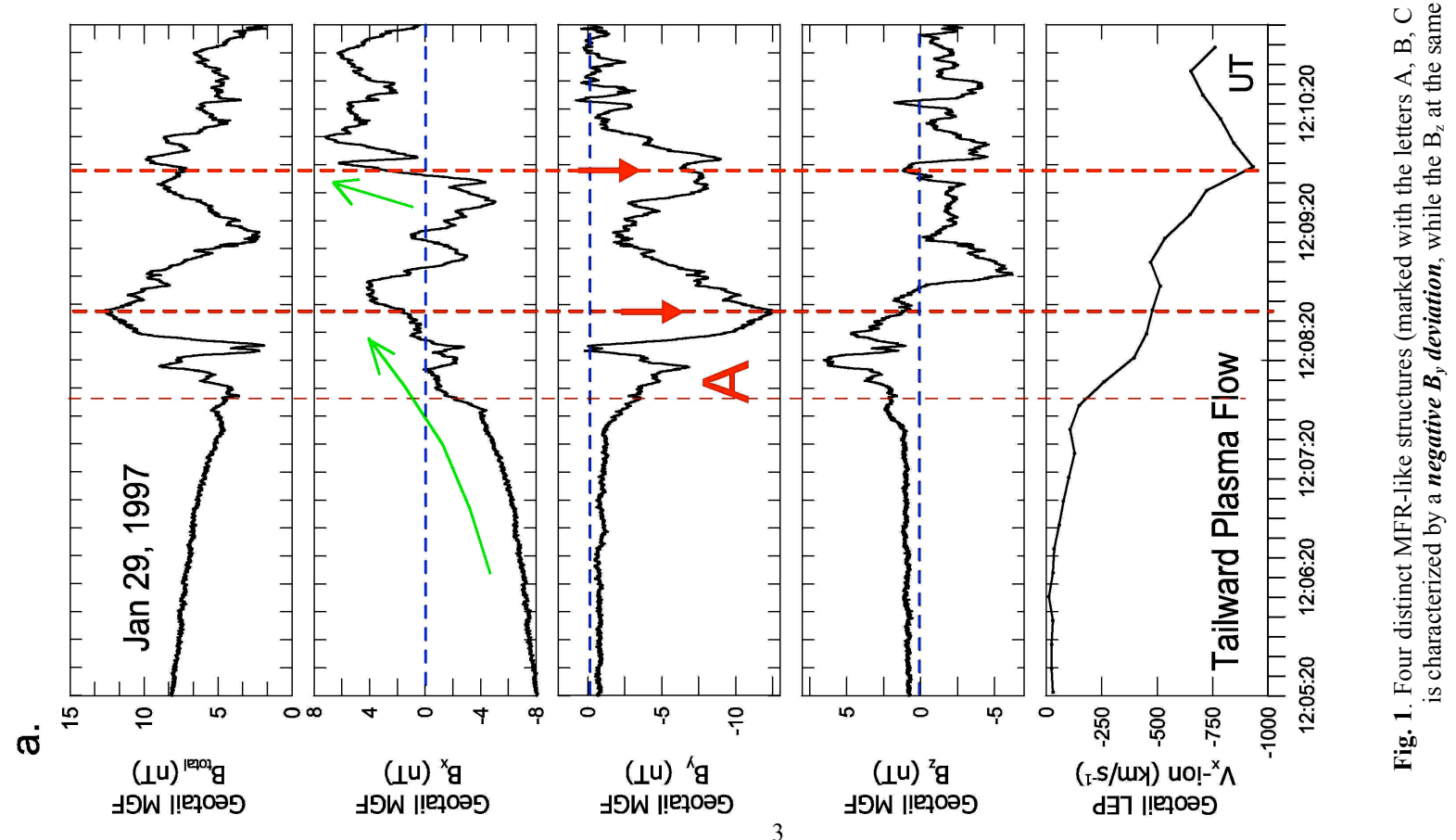



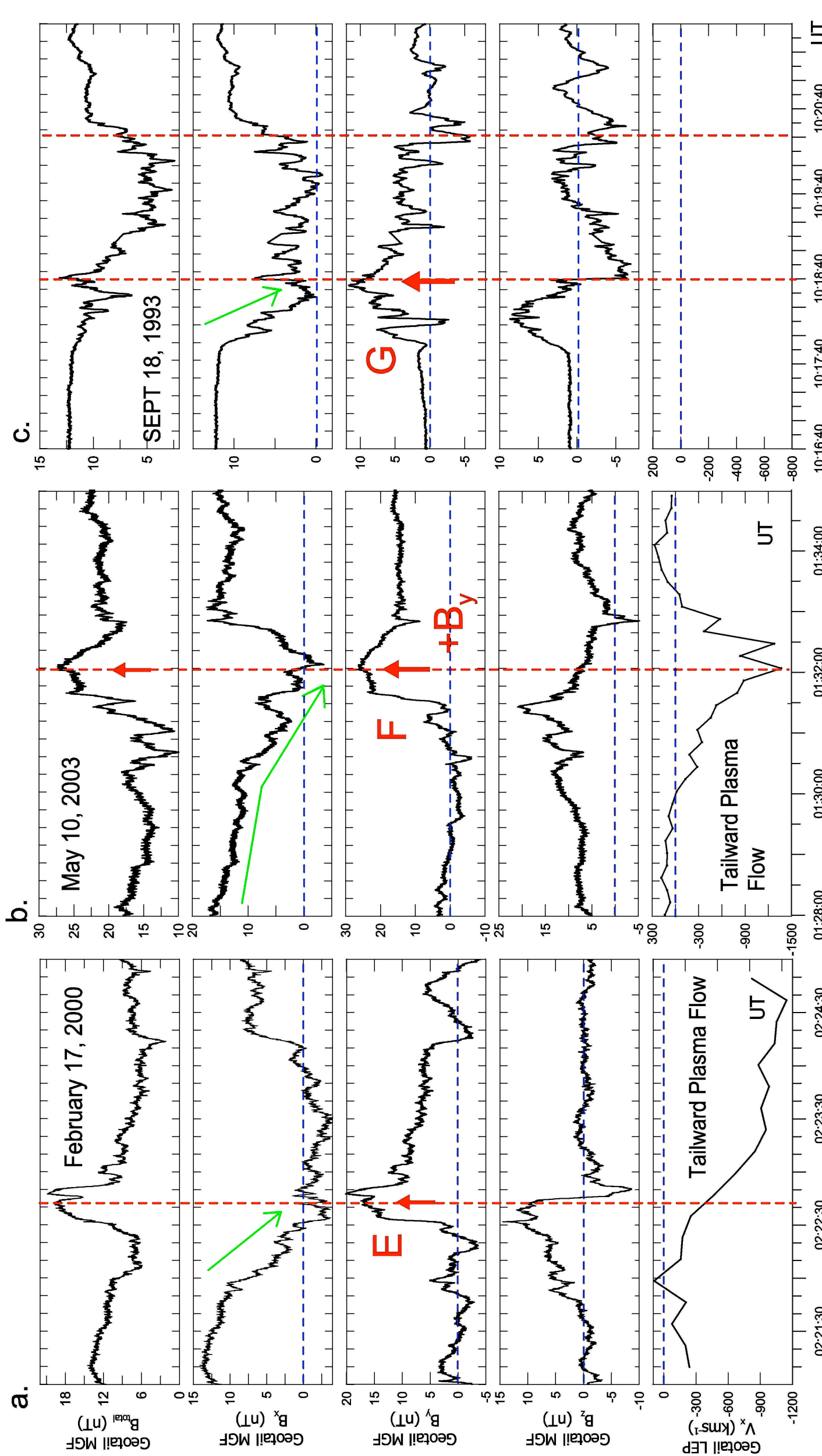

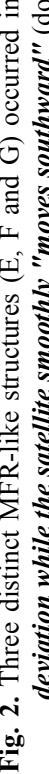




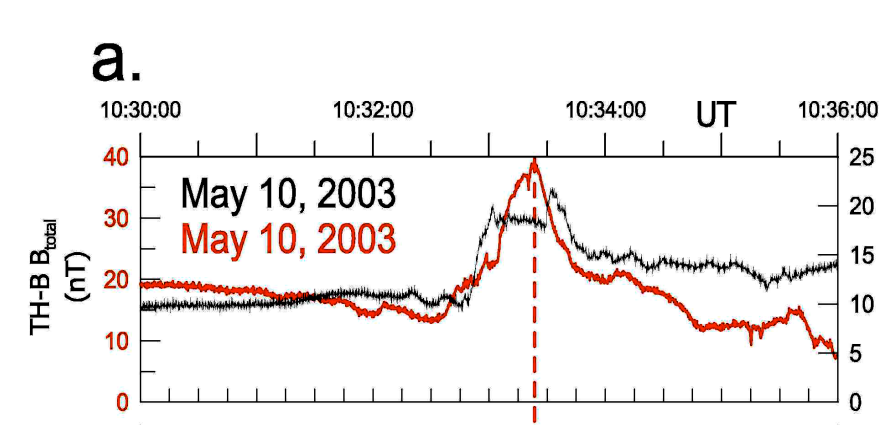

b.
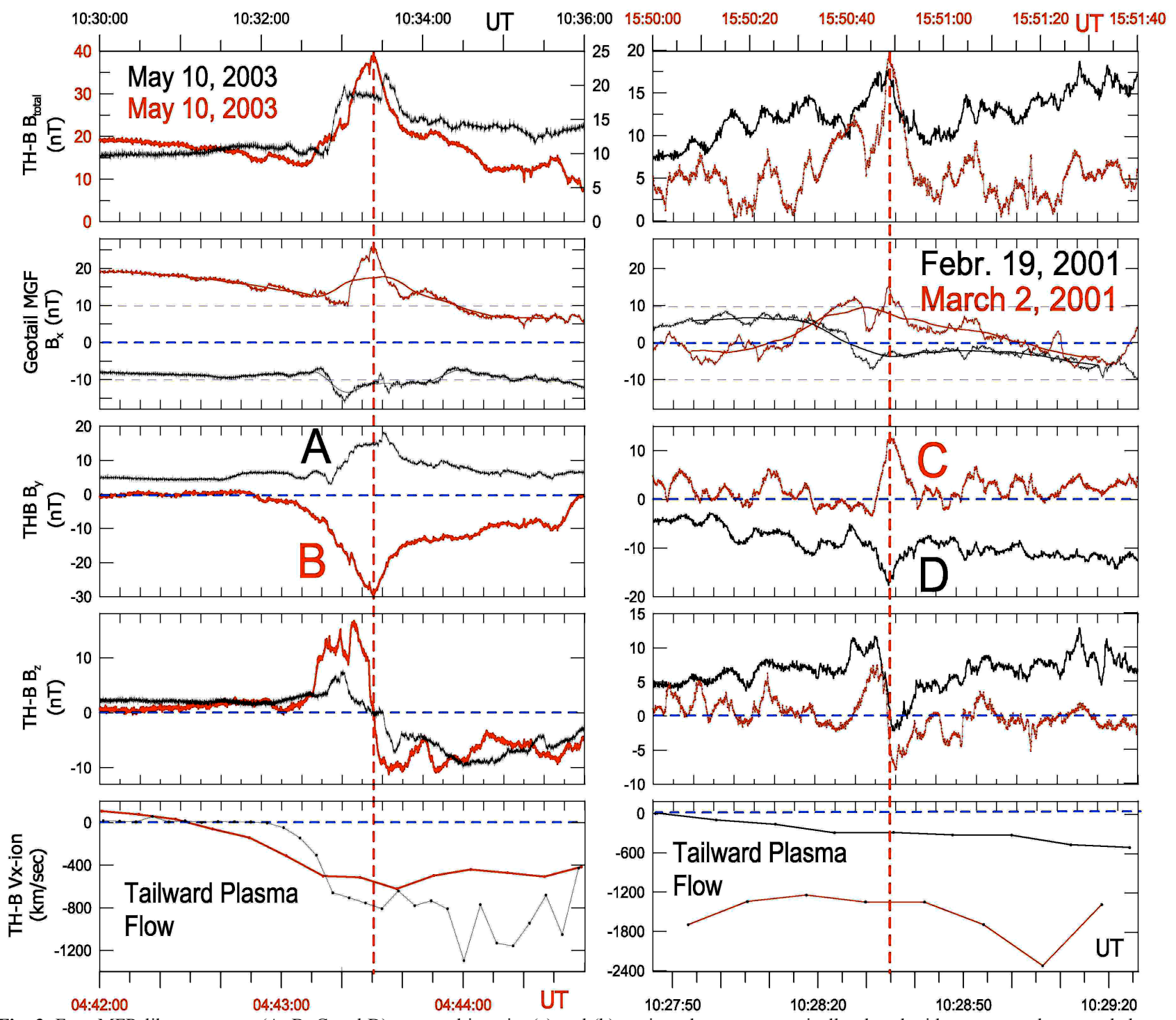

Fig. 3. Four MFR-like structures (A, B, C and D) occurred in pairs (a) and (b) at sites almost symmetrically placed with respect to the neutral sheet plane; outside the CPS region (A and B cases) and inside the CPS (C and D cases). The $\mathrm{B}_{\mathrm{y}}$ deviation outside the CPS is negative (positive) in north (south) plasma sheet, whereas the $\mathrm{B}_{\mathrm{y}}$ inside the CPS is positive (negative) in north (south) plasma sheet.

\subsection{MFRs occurring with an almost motionless magnetotail}

In this subcategory the sign of $\mathrm{B}_{\mathrm{y}}$ deviation depends on the satellite location within the plasma sheet and more specifically from the satellite's distance from the neutral sheet. Certainly a motionless magnetotail is an ideal situation; however, sometimes, this condition corresponds better to the real case-event. The sign of $B_{y}$, in this subcategory, shows an asymmetry with respect to neutral sheet; it is different in north and south low latitudes and it is different between the low and high latitudes for the north (or south) plasma sheet, too. That is, the sign reverses somewhere in between CPS and the region close to the lobes, in the north or south plasma sheet. Typically, in this work the region where the $\left|B_{x}\right|$ is less than $\sim 7 \mathrm{nT}$ (for a satellite positioned 20-30 $R_{E}$ away from the Earth) is defined as CPS; whereas the region adjacent to the lobes is characterized by values for $\left|B_{\mathrm{x}}\right|$ greater than $\sim 10 \mathrm{nT}$. In CPS, the $\boldsymbol{B}_{y}$ is positive at north plasma sheet and negative at south plasma sheet. Adjacent to the lobes the $B_{y}$ signs are reversed; at high latitudes the $B_{y}$ is positive at south plasma sheet and negative at north plasma sheet. Therefore, at least four indicative MFR-like structures are needed to exemplify our suggestion. Figure $3 \boldsymbol{a}$ and $\boldsymbol{b}$ show the $\mathrm{B}_{\mathrm{y}}$ response "at high and low latitudes" of plasma sheet, respectively. It is apparent that actually all the four events show a $\mathrm{B}_{\mathrm{y}}$ deviation tightly accompanied by a bipolar positive-then-negative $B_{z}$ signature, a peaked $B_{\text {total }}$ and tailward ion flow. Adjacent to the lobes, the positive (negative) deviation of $B_{y}$ marked $A(B)$ occurs with a locally average value of $B_{x}$ (thin line) greater than $B_{x} \cong-12$ $\mathrm{nT}(15 \mathrm{nT})$; in contrast, in Fig. 2 the peaked $\mathrm{B}_{\mathrm{y}}$ values occurred around $\mathrm{B}_{\mathrm{x}} \cong 0 \mathrm{nT}$. In CPS, the positive (negative) $B_{y}$ deviation marked $C(D)$ occurs with a local average value (thin line) of $B_{x} \cong 7 n T\left(B_{x} \cong-3 n T\right)$. The $B_{y}$ deviations, in this subcategory of events, marked as A (on May 10, 2003), B (on May 10, 2003), C (on March 2, 2001) and D (on February 19, 2001) occurred at $(\mathrm{X}, \mathrm{Y}, \mathrm{Z})_{\mathrm{GSM}}=(-26,-1.9$, 2.6) $R_{E},(-27.8,0.7,3.8) R_{E},(30.17,0.74,2)$, and $(-25,6.2$, 0.5) $R_{E}$, respectively. It should be underlined that this 
paragraph is related to the behavior of magnetotail at distances 20-30 $\mathrm{R}_{\mathrm{E}}$ far away from Earth.

\subsection{More rope examples associated with the appropriate tail's motions}

We have exhibitzed three examples in subsection 2.1 and three in 2.2. The dates for a few more events with the same behavior (i.e., combining the sign of $\mathrm{B}_{\mathrm{y}}$ deviation with the appropriate type of motion in magnetotail, while the ion flow remains tailward) are merely listed below in the format Day/Month/Year; the reader may further scrutinize these additional events. For the category with positive $\mathbf{B}_{\mathbf{y}}$ deviations we cite the events occurred on (a) 18/02/1999, at 09:40 UT; (b) 11/02/1999, at 14:27 UT; (c) 18/04/2003, at 17:06 UT and (d) 13/03/2003, at 14:05 UT. For the category with negative $\mathbf{B}_{\mathbf{y}}$ deviations we cite the events occurred on (a) $28 / 01 / 1997$, at $13: 35 \mathrm{UT}$; (b) $11 / 02 / 1999$, at 14:32 UT; (c) $11 / 02 / 2000$, at 15:15:30 UT; (d) 08/02/1997, at 16:06:40 UT; (e) 06/03/1999, at 13:45:15 UT; (f) 06/03/1999, at 13:45:10 UT; (g) 14/02/1994, at 14:11 UT; (h) 13/02/1994, at 15:08:40 UT; and (i) 11/12/1994, 17:50-18:20 UT (two cases). Such a repetition mode (based on the same stereotyped observation) can't be expected to occur by shear accident.

\subsection{Extraordinary long-lasting events with intense $B_{y}$ deviation}

In this subcategory, two indicative case studies are selected and presented in Fig. 4a and b; they occurred (a) on May 10 2003 at about 09:35 UT (left-hand panel) and (b) on February 12, 1999, around 02 UT (right-hand panel). In both cases Geotail detected long-lasting $\mathrm{B}_{\mathrm{y}}$ deviations with time life much more than the typical duration of an MFR in magnetotail (i.e., 1-2 min). They occurred at (X, Y, Z) $)_{\mathrm{GSM}}=$ $(-26.3,-1.3,2.8) R_{E}$ and $(-30,5.2,0.3) R_{E}$, respectively. We separately describe the events below, while their interpretation scenario is given at the discussion subsection. Certainly, the big question (posed by these events) is related to the ultimate mechanism producing so long-lived $\mathrm{B}_{\mathrm{y}}$ deviations.

Figure 4a shows an event in which the dominant-positive $\mathrm{B}_{\mathrm{y}}$ deviation (third panel) persists for $\sim 8 \mathrm{~min}$, while the plasma flow is essentially tailward (fifth panel). Very weak positive $B_{z}$ values may precede the $B_{y}$ deviation, whereas negative $B_{z}$ does not follow; in contrast, rather the diametrically opposite behavior is diagnosed. The longlasting $B_{y}$ component stays at a level of $\sim 20 \mathrm{nT}$, while the $B_{x}$ is, from time to time, as small as $5 \mathrm{nT}$; consequently, the satellite is positioned close to the neutral sheet given that the level of the lobe's magnetic field is $\sim 30 \mathrm{nT}$.

Under the hypothesis that we observe an MFR, the Geotail has to receive the tailward plasma flow from an Xline located earthward of it. From the available values of velocity with $12 \mathrm{sec}$ resolution, we estimate the "core length" of the supposed MFR being $\boldsymbol{L}_{\text {core }} \approx 35 \boldsymbol{R}_{\boldsymbol{E}}$, while the portion of structure with positive $B_{z}$ (and tailward flow) has length $\mathrm{L}_{\text {north }} \approx 20 \mathrm{R}_{\mathrm{E}}$. Given that the satellite does not detect the rope's portion with negative $\mathrm{B}_{\mathrm{z}}$, then one may argue that the rope's length is greater than $\sim 55 \mathrm{R}_{\mathrm{E}}$; The Geotail was located at $X=-26.3 R_{E}$. Therefore, the Geotail does not cross an already pre-formed MFR structure; conversely the structure, if any, is going to develop. However, we have to argue that what is observed is irrelevant to any typical MFR; there are two fundamental contradictions. First, any reconnection process should be accompanied by a characteristic negative $B_{z}$ deviation resulting from the rope's helical structure; instead, the observed positive $B_{z}$ values constantly increase as the time elapses. In particular, the onset time of the $\mathrm{B}_{\mathrm{y}}$ deviation is preceded by $\sim 8 \mathrm{~min}$ of tailward plasma flow, produced by an activated reconnection process. From this perspective, the source-satellite traveled distance (on the basis of plasma data) is $\sim 20 \mathrm{R}_{\mathrm{E}}$; and the source-satellite distance practically is $\sim 15 \mathrm{R}_{\mathrm{E}}$. Thus, the onset of the $\mathrm{B}_{\mathrm{y}}$ deviation should be associated with negative $B_{z}$ values, which apparently is not the case. Second, it is illogical that a weak substorm associated with the just studied event can produce such a gigantic MFR releasing tailward an enormous amount of magnetic energy detached from the magnetosphere. We assume that (approximately) the amount of reconnected magnetic field lines should be proportional to the magnetic energy contained in the tailward detached MFR in the same manner as it proportional to the degree of dipolarization of magnetic field earthward of the source. The latter is directly related to the current wedge that is manifested over the ionosphere as the auroral westward electrojet associated with the AL index decreases on the appropriate ground stations within the auroral oval. However, the above hypothesized proportionality is not observationally supported at all. The presented case studies of ropes in Figs. 1-3 suffice to convince us that very smaller MFRs are associated with much more geomagnetically active periods. For instance, the same day of May 10, 2003, we clearly observe two indicative examples with MFRs. The one at $\sim 01: 30$ UT with duration $\sim 90 \mathrm{sec}$ (Fig. 1b) occurred with a peaked AL value $\sim 1200 \mathrm{nT}$ (not shown); the other at $\sim 04: 43$ UT (Fig. 3a) with duration also $\sim 90 \mathrm{sec}$ proportionally occurred with an AL transition from 1700 to $500 \mathrm{nT}$. The presented case in this subsection, at $\sim 09: 35 \mathrm{UT}$, is characterized by an $\mathrm{AL} \approx 250$ $\mathrm{nT}$ and duration $\sim 450 \mathrm{sec}$. Therefore, we conclude that in our case study the $\mathrm{B}_{\mathrm{y}}$ deviation is obviously produced by a filamentary current flowing along the tail.

Figure $4 \mathrm{~b}$ shows one more event in which the dominantpositive $\mathrm{B}_{\mathrm{y}}$ deviation (third panel) persists for $\sim 34 \mathrm{~min}$ (i.e., from $01: 36$ to $02: 10 \mathrm{UT}$ ). The satellite remains in CPS; the $B_{x}$ is less than $10 \mathrm{nT}$, whereas the total magnetic field at lobes is $\sim 30 \mathrm{nT}$. Throughout the whole interval the $\mathrm{B}_{\mathrm{y}}$ is typically greater than $20 \mathrm{nT}$; around 11:55 UT it exceeds the value of $30 \mathrm{nT}$ (being the lobe's value). The plasma ion flow is tailward; consequently, according to the NENL model one should anticipate mainly negative values for $\mathrm{B}_{\mathrm{z}}$. In contrast, we really observe positive $B_{z}$ along with the prevailing $B_{y}$ component. And this event by no means is a typical MFR with cylindrical magnetic field topology extending along the Y-axis; in contrast, one can say that the "core's crosssectional area" must be highly elongated and stretched out covering $\sim 100 \mathrm{R}_{\mathrm{E}}$. 
a.
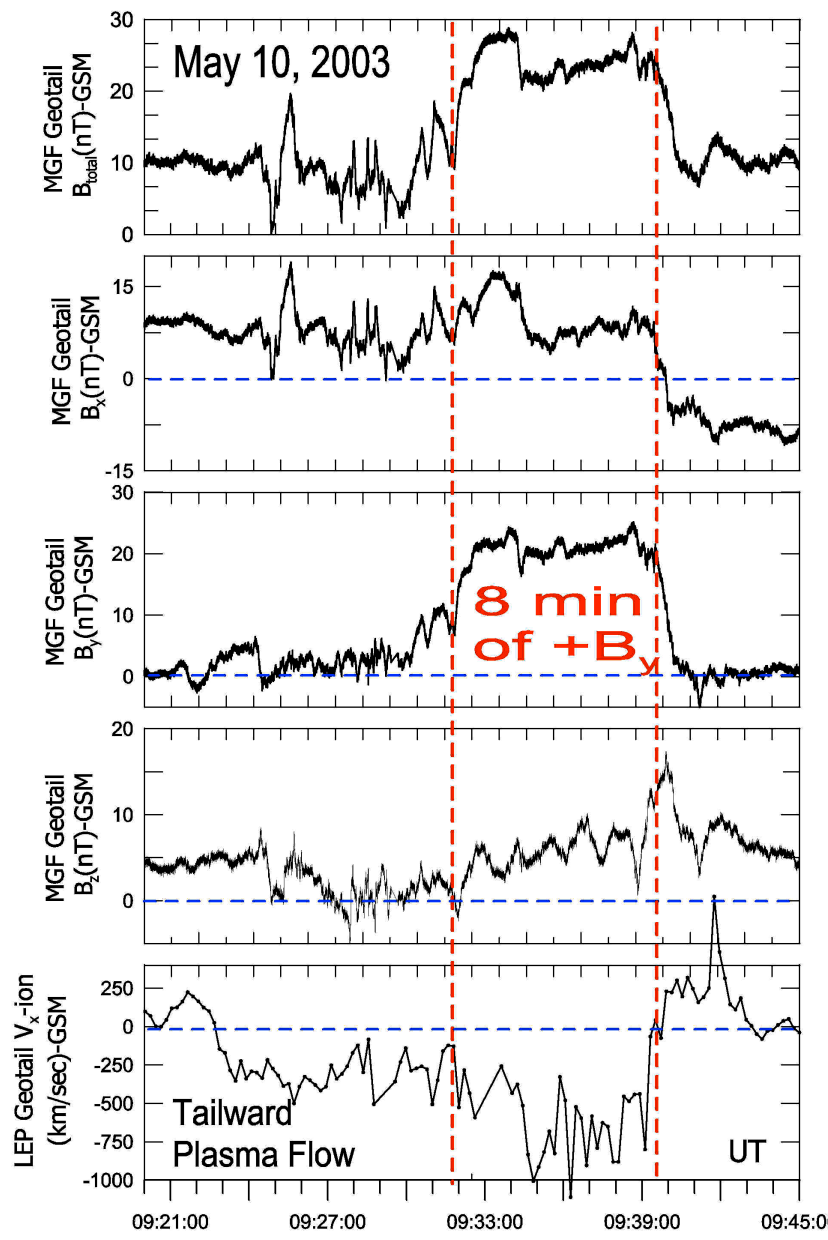

b.

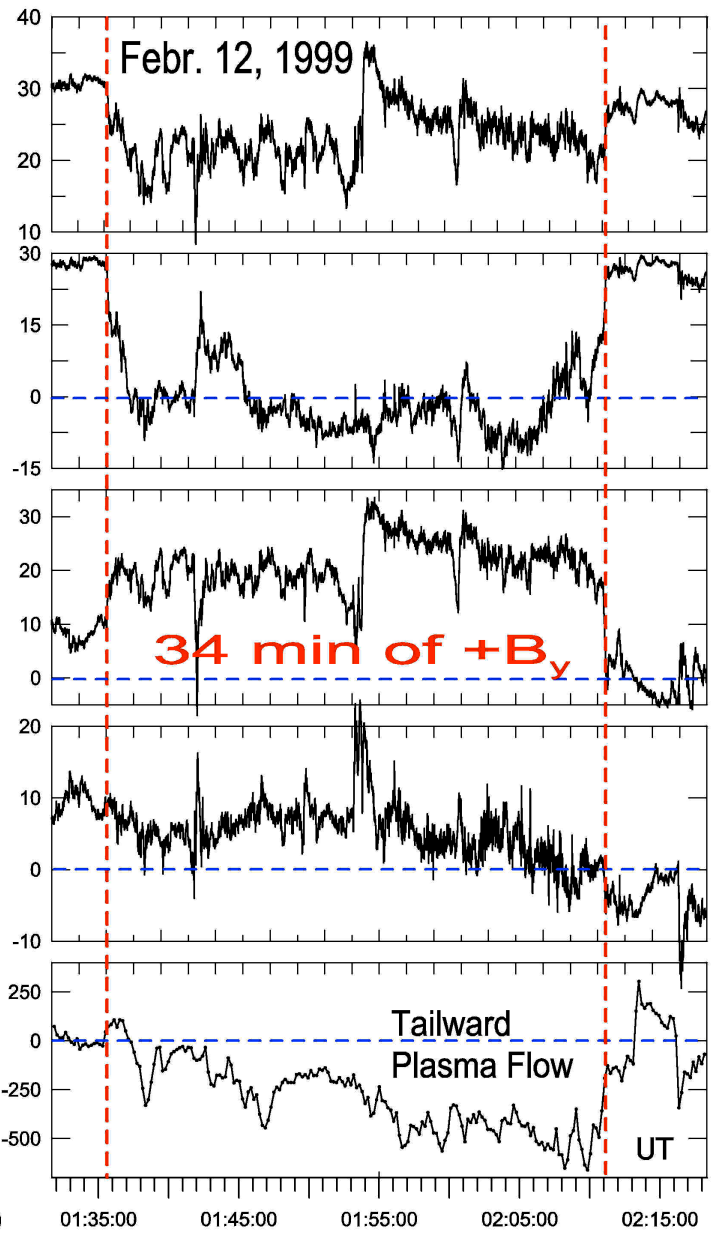

Fig. 4. Extraordinary events (a) and (b) occurred in CPS at 25-30 $\mathrm{R}_{\mathrm{E}}$ far away from the Earth and characterized by long-lasting positive $\mathrm{B}_{\mathrm{y}}$ deviations; the ion-plasma flows are tailward for both events.

\section{Discussion}

3.1. Interpretation scenario of "core's sign" for typical MFR-like structures

3.1.1. Cases associated with vertical plasma sheet excursions:

While the plasma sheet abruptly moves upward or downward (and consequently somewhere locally bends), at that very moment an MFR-like structure occurs; the associated sign with the intense $\mathrm{B}_{\mathrm{y}}$ deviation is essentially determined by the type of motion (i.e., northward or southward). The latter occurs whenever the following three main circumstances are effective and coexist: First, we assume that the plasma sheet is very thin and consequently the ions are demagnetized in a large degree and move tailward within the plasma sheet forming a net "horizontal current" along the tail or "an ion channel" or, generally, "an ion jet". A specific mechanism that potentially produces tailward ion jets is recently introduced by Sarafopoulos [11]. In contrast, the electrons, accomplishing the neutralization process for the jetting ions, maintain their adiabatic motion and basically move "vertically" along the magnetic field lines, while crossing the neutral sheet. Second, in each presented case the satellite first encounters "a positive $B_{z}$ front" usually considered as the rope's tailside-edge of the "helical structure"; within "the front" the $\mathrm{B}_{\mathrm{z}}$ component is the dominant one. In any case, a loop-like magnetic field configuration (over the XZ plane) is probably formed; the so-termed "ion capsule" (by Sarafopoulos, [12, 13]) playing the role of reception region for the tailward jetting ions. Third, a very significant observational element (in this subcategory) is that the tail locally bends upward or downward, when an MFR-like structure occurs, and additionally, the observed ion-plasma velocity steadily increases. Finally, as a consequence of these conditions, while the tailward channeled ions move toward the "ion capsule", at the same time the whole structure is pushed upward or downward, and therefore an ion vortex-like motion is developed within the loop. The clockwise or counterclockwise circulation of ion velocity (producing a cyclic current) finally generates the strong negative or positive $B_{y}$ component identified as the "core" for the MFR-like structure. The ion current flows counterclockwise (clockwise) for an upward (downward) plasma sheet motion, as it is shown in Fig. 5a (5b), respectively. At the capsule's entrance (within a thinned plasma sheet), the ions cross the local magnetic field lines, while at the capsule's interior the ions are primarily switched moving along the lines. The electrons neutralize the positive charge (at the ion propagation front), but there is not a preferential directional flow for electrons along the closed magnetic field lines (i.e., there isn't any electron current crossing the neutral sheet). Apparently, in the context of this work, an MFR-like structure is steadily open at its earthward bottleneck region 
from which an ion current constantly flows in. Therefore, the latter may be an answer to the longstanding question (of paramount importance) concerning the supplier source for the long-lived current feeding the rope and keeping it alive. Any satellite $(\mathrm{S} / \mathrm{C})$ crossing the tailward moving rope, which is asymmetrically placed within the plasma sheet, will inevitably encounter the so-termed $\mathrm{B}_{\mathrm{y}}$-core of rope; such a satellite trajectory is indicated by the blue-dashed line in Fig. 5 .

\subsubsection{Cases associated with locally stationary plasma sheet:}

If the whole magnetotail essentially remains motionless (i.e., the plasma sheet does not bend locally), while a locally thinned plasma sheet together with a loop-like magnetic field structure is developed, then the tailward ejected ions may traverse the almost closed magnetic field structure and form a "two-core" MFR-like configuration, as it is sketched over the meridional-XZ plane in Fig. 5c. Certainly the thickness of the capsule in this case should be comparable to the ion gyro-radius. Even though it sounds like an oxymoron, based on such a topology, the northern (southern) portion of the loop (being the 'ion capsule") is characterized by positive (negative) $\mathrm{B}_{\mathrm{y}}$ deviation; across the loop the $\mathrm{B}_{\mathrm{y}}$ may show a bipolar signature (similar to the situation studied for two events by Sarafopoulos, [7]). Put another way, within the almost closed magnetic field structure of plasma sheet a cross-tail 'magnetic ring" is formed; a torus-like magnetic field like the well-known smoke rings. It is known that a smoke ring is commonly formed when a gust of smoke is suddenly injected into clear air, especially through a narrow opening; the outer parts of the gust are slowed down by the still air (or by edges of the opening) relatively to the central part, imparting it the characteristic poloidal flow pattern. Our magnetic ring structure (seen actually as a ring over the YZ plane) might be detected by simultaneous and vertically separated foursatellite measurements via the appropriately alternating $\pm \mathrm{B}_{\mathrm{y}}$ deviations. In fact, the demand for more than four satellites is imperative in order to reliably estimate the $\Delta \mathrm{Y}$-extent of rope. In this work, given that we are exclusively based on Geotail datasets, we classify the satellite trajectories in four distinct subcategories, on the basis of their distance from the neutral sheet. The satellite distance, in each category, is roughly determined by the $\mathrm{B}_{\mathrm{x}}$ trace-magnitude. The satellite (S/C) trajectories $\operatorname{Tr} 2$ and $\operatorname{Tr} 3$ (Fig. 5c) should be chronologically associated with (a) an intense positive $B_{z}$ deviation, (b) a $\mathrm{B}_{\mathrm{y}}$ deviation positive for $\operatorname{Tr} 2$ and negative for $\operatorname{Tr} 3$ and (c) a $-B_{z}$ deviation. The electrons neutralizing the positive charge will flow along the closed magnetic field lines forming the earthward directed FACs along the upper and lower flanks of the loop-like structure. A satellite trajectory like the $\operatorname{Tr} 1(\operatorname{Tr} 4)$ above (beneath) the loop has to be associated with negative (positive) $\mathrm{B}_{\mathrm{y}}$ deviations. The sign of $\mathrm{B}_{\mathrm{y}}$ is different in CPS and adjacent to the lobe region, either in north or south plasma sheet; in any case the sign of $\mathrm{B}_{\mathrm{y}}$ is dictated by Ampere's law. More specifically, we practically define the typical value of $\left|B_{x}\right|$ to be less than 7 $\mathrm{nT}$ for $\operatorname{Tr} 2$ and $\operatorname{Tr} 3$, and greater than $10 \mathrm{nT}$ for $\operatorname{Tr} 1$ and $\operatorname{Tr} 4$; the satellite is positioned $20-30 \mathrm{R}_{\mathrm{E}}$ away from the Earth.

In this work, the evidence based on single-satellite measurements appeared to be constructive, although merely indicative. In parallel we already know that even with contemporary multi-satellite missions, it is rather impossible to systematically approach this issue. Nevertheless, the concept about an ion current flowing along the tail may be of fundamental importance and we may gain ground in our effort to achieve deeper knowledge for the magnetotail's dynamics. In the MFR cases exhibited in Fig. 3, we stress the fact that each event corresponds to one of the four trajectories of Fig. 5c: (a) The event on May 10, 2003 (Fig. 3a) with $\mathrm{B}_{\mathrm{y}} \cong-28$ and $\boldsymbol{B}_{\boldsymbol{x}} \cong \mathbf{1 3} \boldsymbol{n T}$ corresponds to the $\operatorname{Tr} 1$ track; (b) the event on May 10, 2003 (Fig. 3a) with $\mathrm{B}_{\mathrm{y}} \cong 16$ and $\boldsymbol{B}_{x} \simeq-12 n \boldsymbol{T}$ corresponds to the Tr4 track; (c) the event on March 2, 2001 (Fig. 3b) with $\mathrm{B}_{\mathrm{y}} \cong 20$ and $\boldsymbol{B}_{\boldsymbol{x}} \cong \mathbf{3} \boldsymbol{n T}$ corresponds to the $\operatorname{Tr} 2$ track, and (d) the event on February 19, 2001 (Fig. 3b) with $\mathrm{B}_{\mathrm{y}} \cong-16$ and $\boldsymbol{B}_{\boldsymbol{x}} \cong-\mathbf{3} \boldsymbol{n T}$ corresponds to the $\operatorname{Tr} 3$ track. In conclusion, it can be seen that the proposed schematic of Fig. 5c is under development. Future research might strengthen or disprove the suggested arrangement.

\subsection{On the role of IMF-B}

In the first place, it should be stressed that short-lived $B_{y}$ deviations (inside the plasma sheet) with duration of a few seconds may be fully unrelated to the IMF- $B_{y}$; representative examples in this class of events might be the cases studied by Sarafopoulos using the CLUSTER mission [8]. However, "the large-scale" MFRs, like those shown in Figs. 1 and 2 with an average duration of $\sim 1.5 \mathrm{~min}$, typify the "classic category" of MFRs. It is of crucial importance to discriminate between the two classes, since the MFRs in the second class are probably affected by the IMF- $\mathrm{B}_{\mathrm{y}}$. The intramagnetosphere penetrated IMF- $\mathrm{B}_{\mathrm{y}}$ probably interacts with an already modulated current system in the interior of magnetotail and consequently triggers or accelerates/decelerates an already existing motion. Indeed, for the events of Figs. 1 $a$ and $b$ and $2 a$ the available solar wind datasets (obtained from the ACE and WIND satellites, not shown here) support such a hypothesis: The IMF- $\mathrm{B}_{\mathrm{y}}$ is negative for the events of Fig. $1 \mathrm{a}$ and $\mathrm{b}$ (i.e., -0.8 and $-10 \mathrm{nT}$, respectively), whereas the $\mathrm{IMF}^{-B_{\mathrm{y}}}$ is positive $(\sim 4.5 \mathrm{nT})$ for the event of Fig. 2a. As far as the event of Fig. $2 b$ is considered, there is an uncertainty for the sign of the IMF- $\mathrm{B}_{\mathrm{y}}$ being -2 in GSE and +1 in GSM. If we add a $B_{y}$ component (of exo-magnetosphere origin) over the schematic of Fig. 5c, then we would anticipate that the tail will bend upward

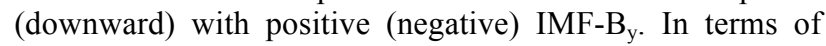
magnetic pressures, such a motion would be due to the spatial asymmetry of pressures, above and below the looplike magnetic field structure. Thus, one can infer that the sign of the IMF- $\mathrm{B}_{\mathrm{y}}$ determines both, the tail's motion around the rope plus the sign of the rope's core. However, we have to make a subtle distinction: First, that the just expressed reasoning is exclusively based on our model introducing "the ion capsule" concept along with its associated FACs. Second, it must be taken seriously into account that "in the reconnection framework of thinking" there is not any demand dictating the tail's motion in relation to the sign of IMF-B $\boldsymbol{B}_{\boldsymbol{y}}$. In any case, the detection of an MFR often coexists with the appropriate tail's motion to the right direction; and this concept is further developed in the next subsection. 


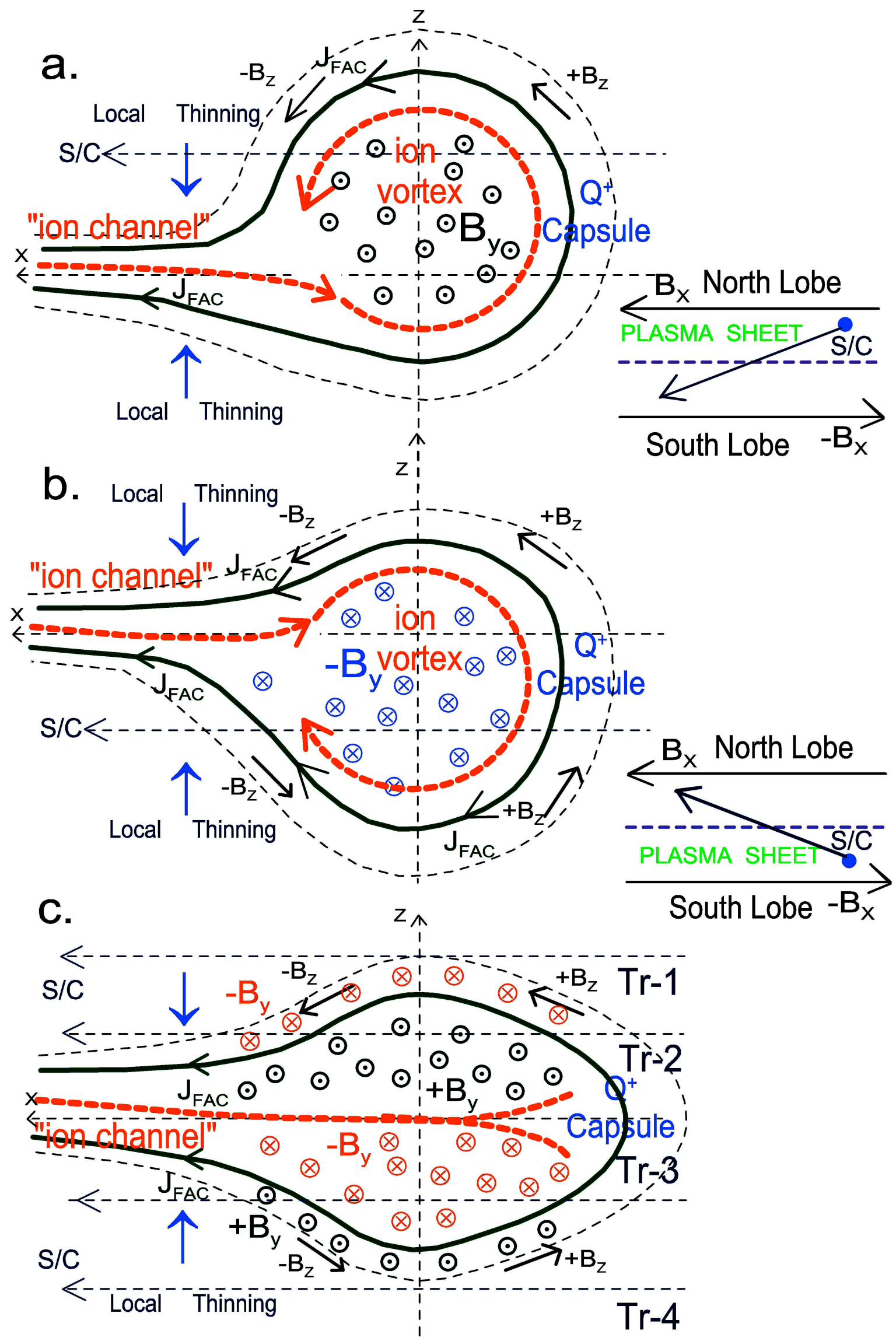

Fig. 5. a) While the tail moves northward (upward) the "core $B_{y}$ " of the MFR-like structure takes positive sign resulting from a counterclockwise flowing ion current (red-dashed line). b) The tail moves southward (downward) and the "core $\mathrm{B}_{\mathrm{y}}$ " of the MFR-like structure (formed by a clockwise flowing ion current) is negative. c) The plasma sheet essentially remains motionless and the $\mathrm{B}_{\mathrm{y}}$ deviation appears positive in north and negative in south plasma sheet; the ions are jetting tailward (like a filamentary current) inside an elongated "ion capsule". 


\subsection{Interpretation scenario for the events with long- lasting $B_{y}$ deviations}

In general, every natural phenomenon should be interpreted by the prevailing theory and always the extraordinary events test in depth any existing model. In parallel with this principle, an additional comment-parameter is worth considering, as we think: In past works sometimes "an ideal MFR structure" is carefully selected and studied (e.g., by Mukai et al., [14]). However, interpreting the "perfect event" in this direction may lurk a danger; we may pre-define the selection criteria and then, obviously, all the events will fulfill our criteria and line up with our own supposed model. For instance, in the distant magnetotail (in parallel to the just mentioned "event of Mukai"), the reader can easily verify our claim that much more complicated Geotail-MFR-like structures really occurred; one may look at the events (a) on February 13, 1994, at 21:01 UT, (b) on February 14, 1994, at 14:11 UT, and (c) on February 15, 1994, at 05:28 UT.

The presented long-lasting events are categorically nontypical MFR events; they are apparently particularly significant. In our understanding, the key process in both cases is that the otherwise (hypothesized) symmetric magnetic field structure around the neutral sheet plane becomes really asymmetric. Accordingly, a question is posed: What is the factor playing such a catalytic role? Below, let us exhibit our procedure of thinking.

First, we begin assuming that within a thinned plasma sheet an ion channel with tailward jetting ions is formed, while the electrons set in the FACs are neutralizing the ion charge. Thus, one may consider that the two branches (north and south) of FACs are initially symmetric (with respect to the neutral sheet plane) and of the same magnitude; the whole configuration looks like to what is already sketched in Fig. 5c. Then, we add the penetrated component of IMF-By, which will catalytically readjust the topology of FACs. The IMF- $\mathrm{B}_{\mathrm{y}}$ is positive in both cases and as large as $\sim 15 \mathrm{nT}$ (for the second event, Fig. 4b). We assume that a portion of the IMF- $B_{y}$ penetrates into the tail and, then, it is added to the $B_{y}$ produced by the FACs. That is, in Fig. $5 \mathrm{c}$, we add a positive component $\mathrm{B}_{\mathrm{y}}$ of exo-magnetosphere origin; therefore, the $B_{y}$ increases below the ion capsule and decreases above it. The overall $\mathrm{B}_{\mathrm{y}}$ interacts with the (local branch of) FAC flowing earthward in north and south plasma sheet alike. Finally, the magnetic force $\mathbf{F}=\mathbf{J}_{\mathrm{FAC}} \mathbf{x} \mathbf{B}_{\mathrm{y}}$ acting over the FACs will push the ensemble of the ion capsule plus a portion of the ion channel upward; the magnetotail locally bends upward as it is shown in Fig. 6. In the modified configuration the jetting ions will encounter the newly formed local curvature (of magnetic field) and, finally, will move "along the magnetic field lines". In this way, the ions will produce a net current encircling the positive "B $\mathrm{B}_{\mathrm{y}}$ core"; a current generated in the southern hemisphere of plasma sheet and flowing to the northern hemisphere. There is a positive feedback between the ever increasing curvature and the magnitude of core leading to the formation of an MFR, an intense $B_{z}$ front and a strong $B_{y}$ core. The 3-D MFR-like structure is not cylindrical at all; instead, its cross sectional area is highly stretched out. The whole configuration looks like as bounded by a system of anti-parallel currents wherein the satellite is positioned. Thus, the events of Fig. 4 could correspond to the sketch of Fig. 6; the Geotail's trajectory is marked as $\operatorname{Tr} 1$.

We stress that the $B_{y}$ deviation inside the plasma sheet should not be directly associated with the IMF- $B_{y}$ penetrated into the plasma sheet; this is clear by the following observation: The IMF- $\mathrm{B}_{\mathrm{y}}$, at least for the event of Fig. $4 \mathrm{~b}$, continues to be equally high and positive after the shown interval, whereas the $\mathrm{B}_{\mathrm{y}}$ values in CPS are insignificant (for instance at about 03:28 UT with tailward ion flow, not shown here). Most importantly, soon after the exhibited interval in Fig. 4b, Geotail again descents (from lobes) into the plasma sheet and stays outside the CPS region, given that the $B_{x}$ is greater than $\sim 10 \mathrm{nT}$, where the $B_{y}$ is about $-7 \mathrm{nT}$ (from 02:40 to 02:50 UT, not shown) and, sometimes, -12 $\mathrm{nT}$, although the IMF- $\mathrm{B}_{\mathrm{y}}$ is as positive as $\sim 15 \mathrm{nT}$. Therefore, at the Geotail site, the influence of a current probably annihilates or even reverses the sign of the penetrated $\mathrm{B}_{\mathrm{y}}$. In our context, this moment the satellite is positioned somewhere along the $\operatorname{Tr} 2$ trajectory (in Fig. 6) along which the $\mathrm{B}_{\mathrm{y}}$ is obviously negative. The latter complies with the observed $B_{x}$ values at that site-time being positive and greater than $\sim 10 \mathrm{nT}$; Geotail is moved toward the north plasma sheet.

Furthermore, a plausible question may be expressed relatively to the preceded paragraph: Which existing reconnection scheme can reconcile the co-existence of very intense positive IMF- $\mathrm{B}_{\mathrm{y}}$ values with a persistently strong and negative $\mathrm{B}_{\mathrm{y}}$ deviation inside the plasma sheet? The present observations underline the fact that the magnetic field alone, without the simultaneous knowledge of currents, does not lead to a correct understanding of the magnetospheric dynamics.

\subsection{A "kink" along the magnetotail}

Typically, well before the satellite encounters the rope's core it moves (northward or southward) approaching the neutral sheet (look at Figs. 1-2). The whole tail bends and the prerope region is characterized by positive $B_{z}$ values. Whenever a satellite crosses such a kink of tail, at high latitudes of plasma sheet close to the lobes, it will detect a travelling compression region (TCR). And under the perspective of this work, the plasmoid/MFR will not be symmetrically placed with respect to the neutral sheet. The latter may be an explanation why the TCRs are, at times, associated with monopolar $-\mathrm{B}_{\mathrm{z}}$ deviations.

\section{Conclusions}

A major observational result is that when (and where) an MFR is detected in magnetotail, the tail itself often moves upward (northward) or downward (southward) and the type of motion turns out to be the critical factor determining the sign for the $\mathrm{B}_{\mathrm{y}}$-core deviation: An upward motion generates a "positive $\mathrm{B}_{\mathrm{y}}$ core", whereas a "negative $\mathrm{B}_{\mathrm{y}}$ core" is generated by a downward motion.

In parallel, extraordinary events within the plasma sheet with long-lasting (up to $34 \mathrm{~min}$ ) and intense $\mathrm{B}_{\mathrm{y}}$ deviation challenge us to give a self-consistent interpretation along with the MFR $\mathrm{B}_{\mathrm{y}}$-type deviations. Toward this direction, we suggest a model in which the key idea is that any "active source" in a thinned plasma sheet (located earthward of the spacecraft) produces an ion jet along the tail; that is, a net "horizontal" and tailward directed current. In contrast, the neutralizing electrons are essentially moving "vertically" along the magnetic field lines and crossing bidirectionally the neutral sheet. Besides the ion jet, if the plasma sheet simultaneously bends, at that very moment the ions will form an ion vortex (projected as a ring over the $\mathrm{XZ}$ plane). 
And this circular current dictates the positive or negative

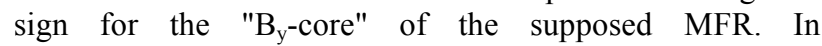
magnetosphere events with long-lasting $\mathrm{B}_{\mathrm{y}}$ deviation, the role of the IMF- $B_{y}$ seems to be of critical importance moving the tail upward (with positive IMF- $\mathrm{B}_{\mathrm{y}}$ ) or downward (with negative IMF- $\mathrm{B}_{\mathrm{y}}$ values). Consequently, the $\mathrm{B}_{\mathrm{y}}$ deviation inside the plasma sheet will be positive for the former case and negative for the latter one. The reason that forces the tail to be locally displaced is analytically explained in our discussion. In summary, several factors have to be considered when determining the sign of the core field in a magnetic flux rope like structure (MFR) embedded in the tailward plasma flow associated with the Earth's magnetotail. Thus, an efficient model has been formulated based on the thickness of the plasma sheet, the front of the $\mathrm{B}_{\mathrm{z}}$-field and the tail bending direction. These conditions together stimulate the formation of an ion vortex and the generation of eddy currents, which define a certain orientation of the core field vector and therefore define its corresponding sign.

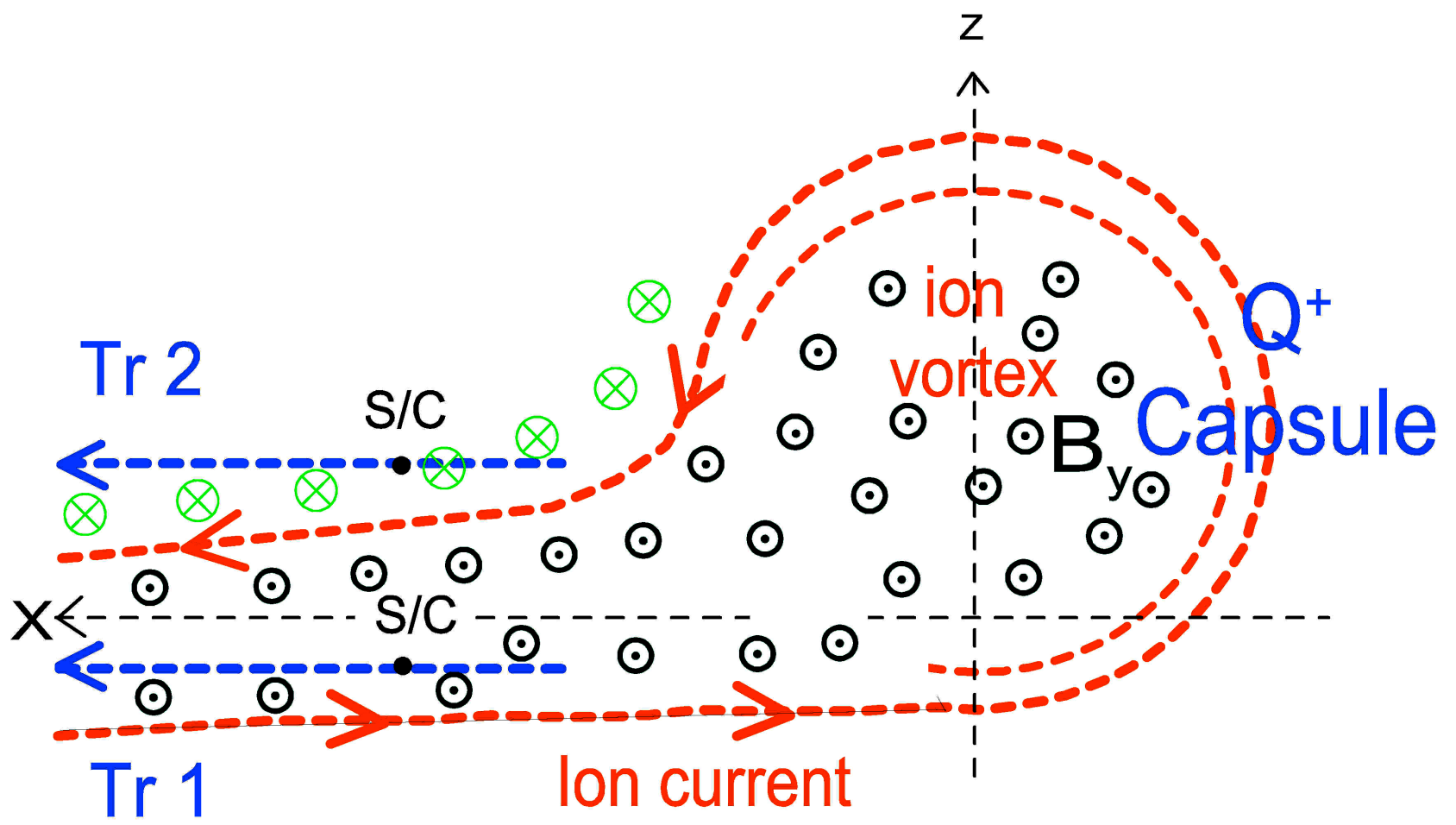

Fig. 6. Likewise to the situation of Fig. $5 \mathrm{a}$, the $\mathrm{B}_{\mathrm{y}}$ deviation is positive and the tail bends upward since the IMF- $\mathrm{B}_{\mathrm{y}}$ is persistently positive. The ion current (red-dashed line) seems to form (for the extraordinary events of Fig. 4) a highly elongated "core-structure". Therefore, the satellite (S/C) spends much time moving along the $\operatorname{Tr} 1$ trajectory placed in a region of almost antiparallel currents; the $\mathrm{B}_{\mathrm{y}}$ deviation is constantly positive.

\section{Acknowledgements.}

We thank Prof. T. Nagai and Yoshifumi Saito for the high resolution Geotail/MGF magnetic field and Geotail/LEP plasma data, respectively. This research has been cofinanced by the European Union (European Social FundESF) and Greek national funds through the Operational Program "Education and Lifelong Learning" of the National
Strategic Reference Framework (NSRF)-Research Funding Program: Thales. Investing in knowledge society through the European Social Fund. The project is called "Hellenic National Network for Space Weather Research" coded as MIS 377274. I thank the persons involved as reviewers.

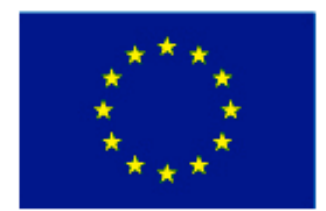

\section{European Union European Social Fund}

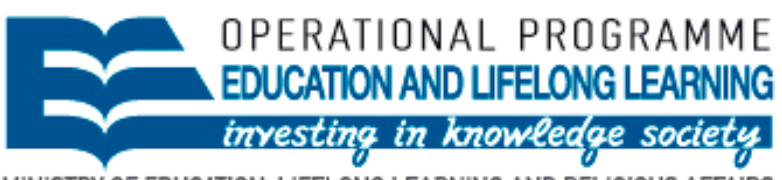

MINISTRY OF EDUCATION. LIFELONG LEARNING AND RELIGIOUS AFFAIRS M A N A G I N G A U T H O R I T Y

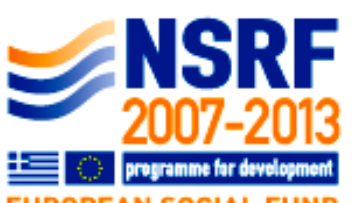

EUROPEAN SOCIAL FUND

\section{Co-financed by Greece and the European Union}

\section{References}

1. Baker, D. N., Pulkkinen, T. I., Angelopoulos, V., Baumjohann, W., and McPherron, R.L.: Neutral line model of substorms: Past results and present view, J. Geophys. Res., 101, 12,975-13010, 1996.
2. Petrukovich, A. A., Origins of plasma sheet $B_{y}$, J. Geophys. Res., 116, A07217, doi:10.1029/2010JA016386, 2011. 
3. Slavin, J. A., Owen, C. J., Kuznetsova, M. M., and Hesse, M.: ISEE 3 observations of plasmoids with flux rope magnetic topologies, Geophys. Res. Lett., 22(15), 2061-2064, 1995.

4. Imber, S. M., J. A. Slavin, H. U. Auster, and V. Angelopoulos.: A THEMIS survey of flux ropes and travelling compression regions: Location of the near Earth reconnection site during solar minimum, J. Geophys. Res., 116, A02201, doi:10.1029/2010JA016026, 2011

5. Slavin, J. A., Lepping, R. P., Gjerloev, J., Fairfield, D. H., Hesse, M., Owen, C. J., Moldwin, M. B., Nagai, T., Ieda, A., and Mukai, T.: Geotail observations of magnetic flux ropes in the plasma sheet J. Geophys. Res., 108(A1), 1015, doi:10.1029/2002JA009557, 2003.

6. Slavin , J. A., Tanskanen, E. I. Hesse, , M., Owen, C. J., Dunlop, M. W., Imber, S., Lucek, E. A., Balogh, A., and Glassmeier, K.H.: Cluster observations of traveling compression regions in the near tail, J. Geophys. Res., 110, A06207, doi:10.1029/2004JA010878, 2005.

7. Sarafopoulos, D. V.: A pseudo-magnetic flux rope observed by the THEMIS satellites in the Earth's magnetotail, Journal of Atmospheric and Solar-Terrestrial Physics, 73, 2279-2288, 2011.

8. Sarafopoulos, D. V.: Substorm-associated dual system of FieldAligned Currents interpreting major bipolar magnetic field signatures in the Earth's magnetotail, Journal of Engineering Science and Technology Review, 6(5), 149-164, 2013

9. Kokubun, S., Yamamoto, T., Acuna, M. H., Hayashi, K., Shiokawa, K., Kawano, H.: The GEOTAIL magnetic field experiment. Journal of Geomagnetism and Geoelectricity 46, 7-21, 1994.

10. Mukai, T., et al.: The low energy particle (LEP) experiment onboard the Geotail satellite. Journal of Geomagnetism and Geoelectricity 46, 669-692, 1994.

11. Sarafopoulos, D. V.: Twin-Double Layer Structure Producing Tailward Ion Jets in the Earth's Magnetosphere, Journal of Engineering Science and Technology Review, 5(2), 76-84, 2012.

12. Sarafopoulos, D. V.: A physical mechanism producing suprathermal populations and initiating substorms in the Earth's magnetotail, Ann. Geophysicae, 26, 1617-1639, 2008.

13. Sarafopoulos, D. V.: Bi-layer structure of counterstreaming energetic electron fluxes: a diagnostic tool of the acceleration mechanism in the Earth's magnetotail, Ann. Geophysicae, 28, 455-477, 2010.

14. Mukai T., Yamamoto T., and Machida S.: Dynamics and kinetic properties of plasmoids and flux ropes: GEOTAIL Observations, Geophysical monograph 105: New perspectives of the Earth's magnetotail, editors A. Nishida, D. Baker and S. Cowley, AGU, 1998. 